\title{
Suppression of APC/CCdh1 has subtype specific biological effects in acute myeloid leukemia
}

\author{
Daniel Ewerth ${ }^{1,2}$, Andrea Schmidts ${ }^{1}$, Manuel Hein ${ }^{1,3}$, Dominik Schnerch ${ }^{1}$, Arunas \\ Kvainickas $^{1,4}$, Christine Greil ${ }^{1}$, Justus Duyster ${ }^{1}$, Monika Engelhardt ${ }^{1}$, Ralph Wäsch ${ }^{1}$ \\ ${ }^{1}$ Department of Hematology, Oncology and Stem Cell Transplantation, University Medical Center, Faculty of Medicine, \\ University of Freiburg, Freiburg, Germany \\ ${ }^{2}$ Faculty of Biology, University of Freiburg, Freiburg, Germany \\ ${ }^{3}$ Present address: Department of Cardiology and Angiology II, University Heart Center Freiburg - Bad Krozingen, Faculty of \\ Medicine, University of Freiburg, Bad Krozingen, Germany \\ ${ }^{4}$ Present address: Center for Biological Systems Analysis, University of Freiburg, Freiburg, Germany \\ Correspondence to: Ralph Wäsch, email: ralph.waesch@uniklinik-freiburg.de \\ Keywords: anaphase-promoting complex, Cdhl, ubiquitin-ligase, acute myeloid leukemia, differentiation \\ Received: September 06, $2015 \quad$ Accepted: June 09,2016 Published: June 21, 2016
}

\section{ABSTRACT}

The E3 ubiquitin ligase and tumor suppressor APC/ $C^{\text {cdh1 }}$ is crucial for cell cycle progression, development and differentiation in many cell types. However, little is known about the role of Cdh1 in hematopoiesis. Here we analyzed Cdh1 expression and function in malignant hematopoiesis. We found a significant decrease of Cdh1 in primary acute myeloid leukemia (AML) blasts compared to normal CD34+ cells. Thus, according to its important role in connecting cell cycle exit and differentiation, decreased expression of Cdh1 may be a mechanism contributing to the differentiation block in leukemogenesis. Indeed, knockdown (kd) of Cdh1 in HL-60 cell line (AML with maturation, FAB M2) led to less differentiated cells and a delay in PMA-induced differentiation. Acute promyelocytic leukemia (APL, FAB M3) is an AML subtype which is highly vulnerable to differentiation therapy with all-trans retinoic acid (ATRA). Accordingly, we found that APL is resistant to a Cdh1-kd mediated differentiation block. However, further depletion of Cdh1 in APL significantly reduced viability of leukemia cells upon ATRA-induced differentiation. Thus, low Cdh1 expression may be important in AML biology by contributing to the differentiation block and response to therapy depending on differences in the microenvironment and the additional genetic background.

\section{INTRODUCTION}

In the hematopoietic system balance between cell cycle progression on the one hand, and cell differentiation preceded by cell cycle exit on the other hand, is vital. Moreover, cell cycle control may be a reasonable target in acute myeloid leukemia (AML) $[1,2]$. The anaphasepromoting complex/cyclosome (APC/C) is an E3 ubiquitin ligase that governs the cell cycle by targeting numerous cell cycle regulators for proteasomal destruction. Its coactivator Cdh1 is needed to establish a stable G0/G1 phase, which is an important precondition for precise cell cycle progression or differentiation and maintenance of genomic stability [3-8]. Thus, loss of Cdh1 may contribute to tumorigenesis by enhanced proliferation of undifferentiated and genetically unstable cells [9].

It has been shown in various models that APC/ $\mathrm{C}^{\text {Cdh1 } 1}$ establishes a stable $\mathrm{G} 1 / \mathrm{G} 0$ phase by maintaining a low mitotic cyclin state [10-13] and degrading the $\mathrm{F}$ box protein Skp2, which leads to the stabilization of the $\mathrm{SCF}^{\text {Skp2 }}$ targets and Cdk inhibitors p21 and p27 $[14,15]$. In contrast, conditional inactivation of $\mathrm{APC} / \mathrm{C}$ function causes quiescent $\mathrm{G} 1 / \mathrm{G} 0$ mouse hepatocytes to re-enter the cell cycle [16]. APC/C $\mathrm{C}^{\mathrm{Cdh} 1}$ also modulates TGF $\beta$ signaling by degrading the transcriptional regulators Klf4 and SnoN to induce target gene expression, which regulates growth 
inhibition and cell differentiation [17-19]. Other important $\mathrm{APC} / \mathrm{C}^{\mathrm{Cdh} 1}$ targets to control the differentiation process are Id (inhibitor of differentiation) proteins [8].

A role of $\mathrm{APC} / \mathrm{C}^{\mathrm{Cdh} 1}$ in the differentiation process has already been described in several cell types, such as neurons, myocytes, lens epithelial cells, hepatocytes and embryonic stem cells [16, 20-24]. However, little is known about the role of Cdh1 in the hematopoietic system. In order to study the role of $\mathrm{APC} / \mathrm{C}^{\mathrm{Cdh}} 1$ in $\mathrm{AML}$, we analyzed the protein expression patterns of $\mathrm{Cdh} 1$ in primary human AML blasts and the role of Cdh1 knockdown $(\mathrm{kd})$ on induced differentiation in two cell lines derived from different AML subtypes using our previously validated highly efficient short hairpin (sh)RNA against Cdh1 [4, 25]. Cdh1 expression was decreased in the vast majority of primary AML samples. Further Cdh1 depletion contributed to a differentiation block in AML with maturation (FAB M2). On the contrary, acute promyelocytic leukemia (APL, FAB M3) with the unique $\mathrm{t}(15 ; 17)$ translocation, where ATRA-induced differentiation is a highly efficient targeted treatment approach, was resistant to the Cdh1-kd effect on differentiation. However, viability of APL cells upon ATRA treatment was significantly reduced.

\section{RESULTS}

\section{Cdh1 expression in primary AML samples}

We examined Cdh1 expression levels in 29 samples of newly diagnosed AML patients. The leukemic blasts analyzed were obtained both from bone marrow (BM; 17/29) and peripheral blood (PB; 12/29) (Table 1). Except for one, primary AML cells showed a strong decrease of Cdh1 in all samples compared to normal PB CD34+ control samples (Figure $1 \mathrm{~A}-1 \mathrm{C}, \mathrm{p}<0.001$ ). In 4 of the samples (\#18, \#21, \#20,\#15), this decrease was greater than 10-fold (Figure 1A). The decrease of Cdh1 expression was similar in blasts from $\mathrm{BM}$ and $\mathrm{PB}$. No correlation between patient data, such as age, gender, cytogenetics, mutations, or FAB subtype and Cdh1 expression could be detected (Table 1). We also analyzed the Cdh1 expression of AML cell lines NB4 and HL-60 and found that Cdh1 in both AML cell lines was much lower expressed and about half of what we observed in PB CD34+ control samples (Figure 1D, 1E).Therefore, we confirmed that the cell lines were comparable to primary samples.

To further investigate the low abundance of Cdh1 in primary AML cells, we reanalyzed published microarray data [26]. Results showed that Cdh1 transcription levels were not significantly different in CD34+ AML cells compared to normal CD34+ cells (Figure 1F). These findings denote that decreased Cdh1 protein expression in primary AML blasts is predominantly due to a posttranscriptional mechanism, such as gene silencing by specific microRNAs or induction of protein degradation. Indeed proteolysis of Cdh1 mediated by the ubiquitin- ligase SCF has been described in human cancer cell lines $[27,28]$. Therefore, we inhibited the SCF complex by expressing a dominant-negative mutant of the core SCF subunit Cullin-1 (Cull-N252) and found a strong increase of Cdh1 in the AML cell lines Kasumi-1 and HL-60, while there was no difference in the cell cycle profile (Figure 1G). This is consistent with SCF-dependent post-transcriptional degradation of $\mathrm{Cdh} 1$ as a potential underlying mechanism of low Cdh1 expression in AML. $\mathrm{F}$ box proteins mediate target specificity of the SCF ubiquitin ligases and it has been demonstrated that the $\mathrm{F}$ box protein Skp2 is frequently increased in AML [29]. Thus, elevated expression of Skp2 could be responsible for low Cdh1 expression in AML. Accordingly, we found increased Cdh1 expression upon Skp2-kd in HL-60 and NB4 myeloid leukemia cells (Figure 2A, Figure 4A). Therefore, we also analyzed the published microarray data set [26] for Skp2 expression and found a slightly lower expression of Skp2 in primary AML. Thus, the underlying mechanism of the described increase of Skp2 protein [29] may also be post-transcriptional.

\section{Cdh1 knockdown contributes to a differentiation block in HL-60}

To evaluate the functional role of Cdh1 in AML, we performed knockdown (kd) experiments using the FAB M2 cell line HL-60 [30]. Cdh1-kd led to stabilization of its target protein Skp2 with corresponding slight decrease of p27 indicating functional knockdown (Figure 2A). In contrast, the kd of Skp2 led to stabilization of its target protein p27 and interestingly of Cdh1. There was also a significant decrease of CD11b expression in Cdh1-kd cells compared to shGFP or shSkp2 suggesting a less differentiated state or inhibition of maturation (Figure 2B).

Neither Cdh1-kd nor Skp-kd had an effect on the cell cycle profile of HL-60 cells (Figure 2C). Next, we stimulated HL-60 cells with phorbol-12-myristate-13acetate (PMA) to induce differentiation. Without PMA stimulation the significant difference in CD11b expression remained stable over 48 hours (Figure 2D). In addition the Cdh1 target proteins Skp2 and Id2 also remained stabilized over 48 hours (Figure 2E). When using low doses of PMA at $1 \mathrm{nM}$, cells differentiated over time with and without Cdh1-kd, but the significant lower level of CD11b expression in cells depleted of Cdh1 was preserved (Figure 2F). The maximum CD11b expression was reached at $24 \mathrm{~h}$ of 1 or $50 \mathrm{nM}$ PMA stimulation. CD14 was slightly induced on HL-60 cells upon $1 \mathrm{nM}$ PMA stimulation for $24 \mathrm{~h}$ with a diminished induction due to Cdh1-kd. CD34 was only marginally expressed on HL-60 +/- Cdh1-kd with and without PMA stimulation (Supplementary Figure S2A). At the protein level the Cdh1 targets were still more stabilized upon Cdh1-kd as compared to the control, but PMA stimulation led to 
Table 1: Patient characteristics

\begin{tabular}{|c|c|c|c|c|c|c|c|}
\hline Patients & Age (Years) & Sex & FAB & Source & Blasts (\%) & Cytogenetics & Mutations \\
\hline AML\#1 & 76 & $\mathrm{~F}$ & M1 & $\mathrm{PB}$ & 97 & normal & \\
\hline AML\#2 & 26 & $\mathrm{~F}$ & M1 & PB & 86 & complex & \\
\hline AML\#3 & 63 & $\mathrm{~F}$ & M1 & PB & 85 & normal & $\begin{array}{c}\text { FLT-LM, } \\
\text { NPM1 }\end{array}$ \\
\hline AML\#4 & 65 & M & M1 & PB & 92 & normal & \\
\hline AML\#5 & 21 & M & $\mathrm{M} 1 / 2$ & PB & 99 & +11 & \\
\hline AML \#6 & 60 & $\mathrm{~F}$ & M2 & PB & 67 & $\mathrm{t}(8 ; 21)$ & \\
\hline AML\#7 & 67 & M & M2 & $\mathrm{BM}$ & 85 & inv 16 & \\
\hline AML\#8 & 19 & M & M3 & $\mathrm{BM}$ & 80 & $\mathrm{t}(15 ; 17)$ & \\
\hline AML\#9 & 63 & $\mathrm{~F}$ & M3 & $\mathrm{BM}$ & 95 & $\mathrm{t}(15 ; 17)$ & FLT-LM \\
\hline AML\#10 & 58 & $\mathrm{~F}$ & M3 & $\mathrm{PB}$ & 88 & $\mathrm{t}(15 ; 17),+8$ & \\
\hline AML\#11 & 63 & M & M3 & $\mathrm{BM}$ & 99 & $\mathrm{t}(15 ; 17)$ & \\
\hline AML\#12 & 36 & M & M4eo & $\mathrm{BM}$ & 99 & normal & FLT-LM \\
\hline AML\#13 & 28 & $\mathrm{~F}$ & M4eo & $\mathrm{BM}$ & 90 & inv16 & \\
\hline AML\#14 & 46 & $\mathrm{~F}$ & M4eo & $\mathrm{BM}$ & 70 & $\begin{array}{l}\text { inv16, 7q-, } \\
\text { complex }\end{array}$ & \\
\hline AML\#15 & 58 & M & tAML & $\mathrm{BM}$ & 65 & $-Y$ & \\
\hline AML\#16 & 31 & M & $\mathrm{M} 4 / 5$ & $\mathrm{BM}$ & 99 & normal & \\
\hline AML\#17 & 74 & M & $\mathrm{M} 4 / 5$ & PB & 99 & $20 q-$ & NPM1 \\
\hline AML\#18 & 61 & M & $\mathrm{M} 4 / 5$ & $\mathrm{BM}$ & 90 & normal & \\
\hline AML\#19 & 70 & $\mathrm{~F}$ & M5 & $\mathrm{BM}$ & 99 & $9 q-$ & $\begin{array}{c}\text { FLT-TKD, } \\
\text { NPM1 }\end{array}$ \\
\hline AML\#20 & 51 & $\mathrm{~F}$ & M5 & $\mathrm{BM}$ & 55 & $+14, \mathrm{t}(1 ; 3)$ & FLT3-LM \\
\hline AML\#21 & 56 & $\mathrm{~F}$ & M5 & $\mathrm{BM}$ & 90 & normal & NPM1 \\
\hline AML\#22 & 66 & F & M5 & PB & 80 & normal & CBL, NPM1 \\
\hline AML\#23 & 68 & F & M5 & PB & 95 & $\mathrm{t}(11 ; 19)$ & \\
\hline AML\#24 & 82 & $\mathrm{~F}$ & AML/MDS & $\mathrm{BM}$ & 55 & normal & RUNX1 \\
\hline AML\#25 & 64 & M & NOS & $\mathrm{BM}$ & 99 & normal & $\begin{array}{l}\text { FLT3-LM, } \\
\text { NPM1 }\end{array}$ \\
\hline AML\#26 & 66 & $\mathrm{~F}$ & NOS & $\mathrm{BM}$ & 99 & normal & $\begin{array}{l}\text { FLT-LM, } \\
\text { NPM1 }\end{array}$ \\
\hline AML\#27 & 67 & M & NOS & PB & 80 & $\mathrm{t}(6 ; 9)$ & FLT-LM \\
\hline AML\#28 & 31 & M & NOS & $\mathrm{BM}$ & 99 & normal & \\
\hline AML\#29 & 46 & F & M4 & PB & 95 & normal & $\begin{array}{c}\text { FLT-LM, } \\
\text { NPM1 }\end{array}$ \\
\hline
\end{tabular}

F: female, M: male

FAB: French-American-British classification, NOS: not otherwise specified, MDS: myelodysplastic syndrome BM: bone marrow, PB: peripheral blood 
increased Cdh1 expression and a decline of the targets over time in line with increased differentiation (Figure $2 \mathrm{G})$. When stimulated with high concentrations of $50 \mathrm{nM}$ PMA, cells rapidly reached maximal CD11b- expression after 24 hours irrespective of Cdh1-kd. The initial lower CD11b level due to Cdh1-kd disappeared (Figure 2H) and Cdh1 protein expression increased with reciprocal decline of its targets (Figure 2I). Our data suggest that low Cdh1 expression contributes to the differentiation block in AML. However, stimulation with high concentrations of PMA $(50 \mathrm{nM})$ led to re-expression of Cdh1, most likely due to enforced transcription of Cdh1 counterbalancing RNAi, which led to consecutive differentiation.

\section{Stimulation of NB4 cells with ATRA led to differentiation and cell cycle arrest}

Targeted differentiation therapy with all-trans retinoic acid (ATRA) is highly effective in acute promyelocytic leukemia (APL) with the $t(15 ; 17)$ translocation. Since we found low expression of $\mathrm{Cdh} 1$ also in APL patient samples (\#13, \#15, \#17, \#23; Table 1, Figure 1A), we investigated the role of Cdh1 in ATRAinduced differentiation in APL. Stimulation of the APL cell line NB4 with $1 \mu \mathrm{M}$ ATRA led to strong increase of CD11b at 24 hours (Figure 3A) and granulocytic differentiation over time (Figure 3B). ATRA stimulation also caused gradual reduction of Skp2 expression until complete loss
A
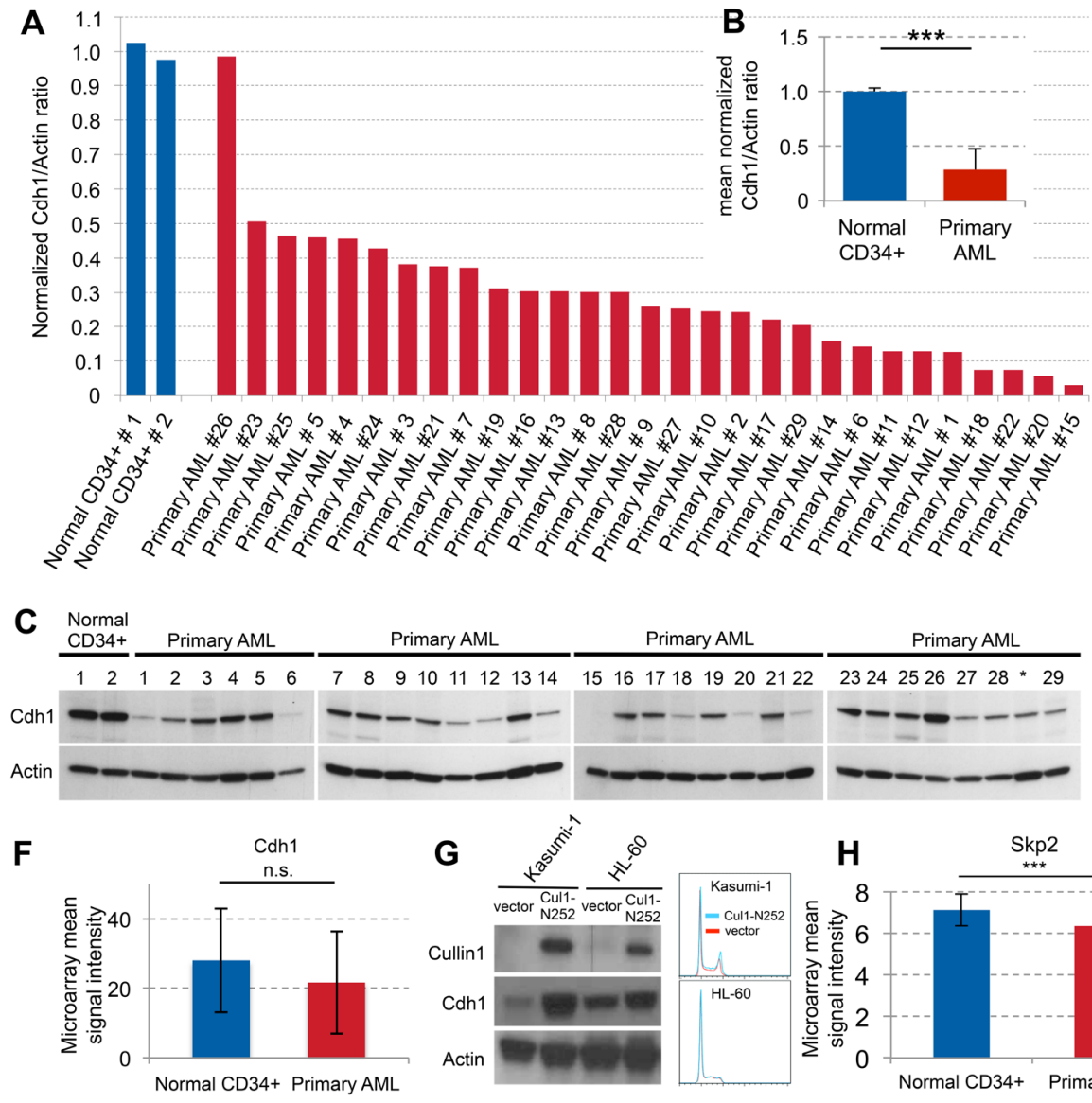

imary AML

Primary AML
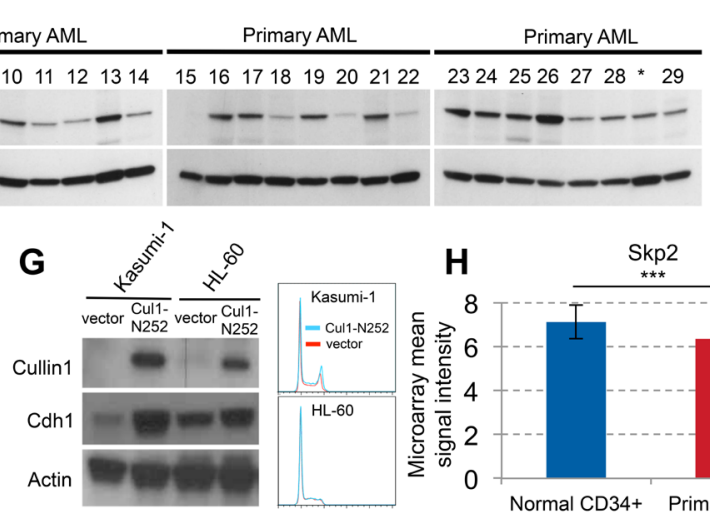

D

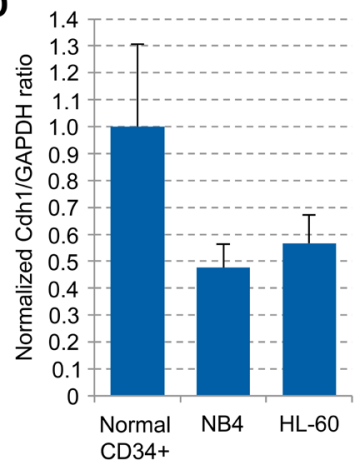

E

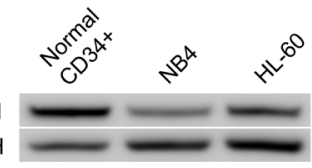

Figure 1: Cdh1 expression in primary AML samples and regulation in cell lines. A. Normal CD34+ cells and samples from 29 AML patients were analyzed by western blot. Quantification of protein expression was used to determine Cdh1/Actin ratio and results were normalized to the mean of the 2 normal CD34+ samples. B. Normalized Cdh1/Actin ratio of primary AML samples presented as mean + s.d. $\mathrm{p}<0.001$. C. Immunoblots for the indicated proteins as quantitated in (A and B). * Sample was excluded due to low blast count. D. Normal CD34+ cells and the AML cell lines NB4 and HL-60 were analyzed by western blot. Quantification of protein expression was used to determine Cdh1/GAPDH ratio and results were normalized to the mean of 3 different normal CD34+ samples. E. Immunoblots for the indicated proteins as quantitated in (D). F. Transcriptional analysis of Cdh1 expression in normal CD34+ and primary AML cells based on publicly available microarray dataset presented as mean $+/-$ s.d. (GSE30029) [26]. n.s. - not significant. G. AML cell lines Kasumi-1 and HL-60 were lentivirally transduced with a construct to overexpress truncated Cullin 1 (Cul1-N252) or with empty vector, respectively and immunoblotted for the indicated proteins. Cell cycle histogramms are shown on the right. H. Transcriptional analysis of Skp2 expression in normal CD34+ and primary AML cells based on publicly available microarray dataset presented as mean +/- s.d. (GSE30029) [26]. *** $\mathrm{p}<0.001$. 
A

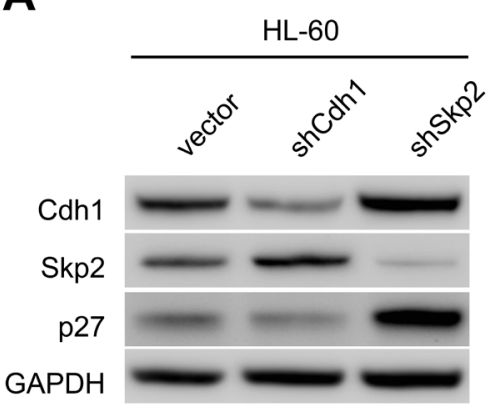

B

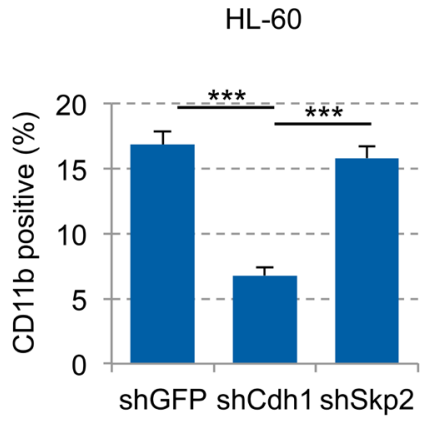

C

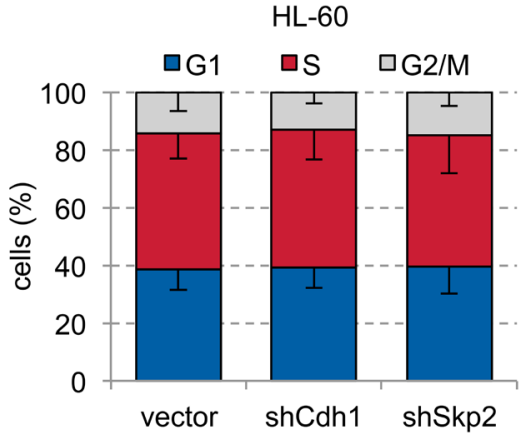

D

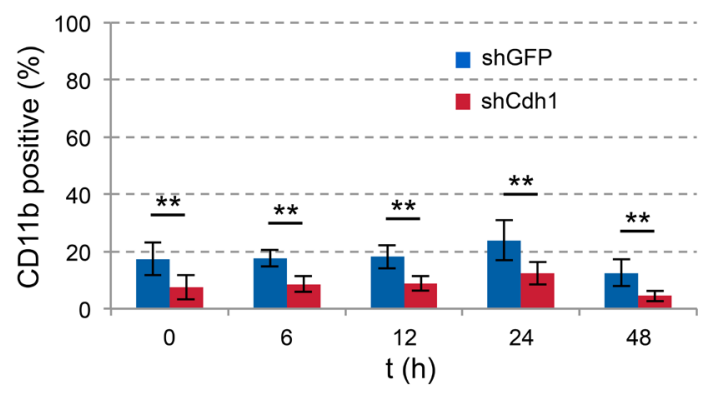

F

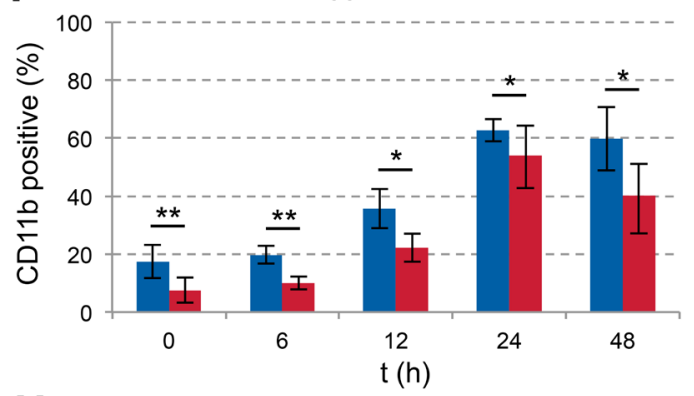

H

HL-60 50 nM PMA

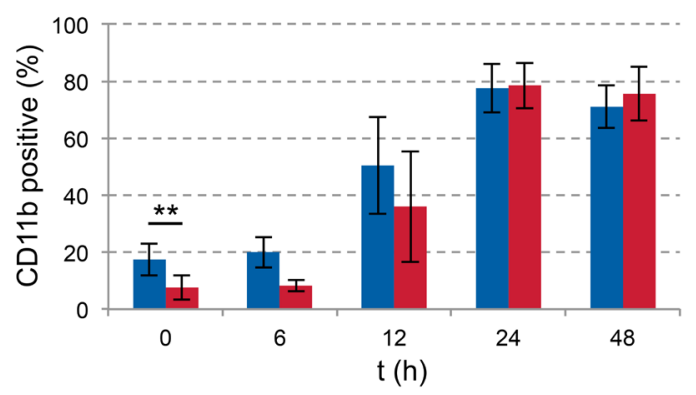

E

HL-60 0 nM PMA

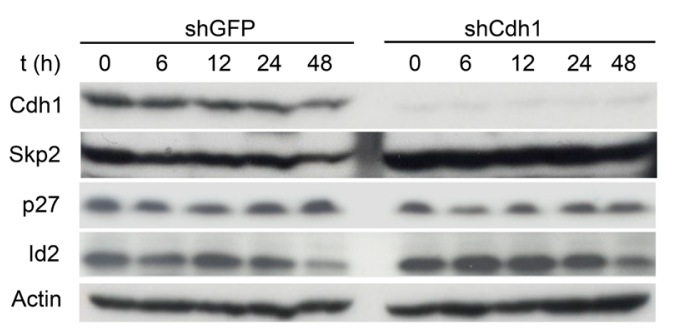

G

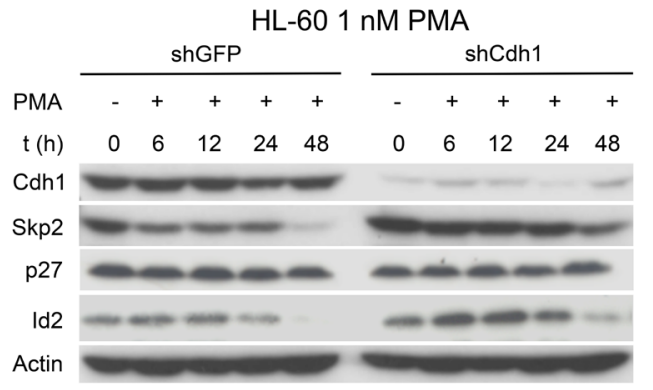

I

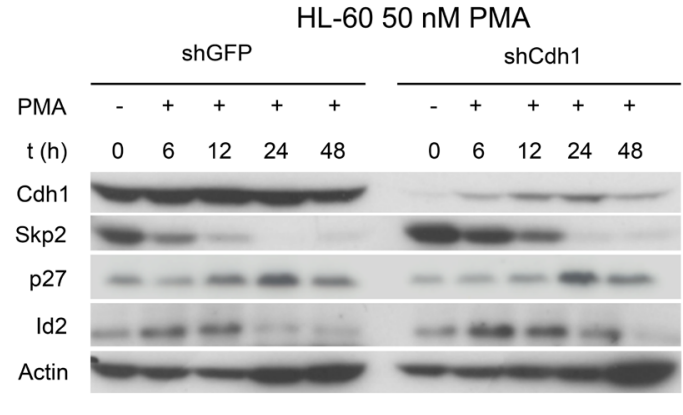

Figure 2: Influence of Cdh1-kd in AML cell line HL-60 on PMA-induced monocytic differentiation. A. HL-60 cells were transduced with vectors, expressing shRNA against human Cdh1 or Skp2 mRNA and analyzed by western blot for indicated proteins. B. Flow cytometric detection of CD11b expression in transduced HL-60 cells presented as mean $+\mathrm{s}$. d. $(\mathrm{n}=3)$; $* * * \mathrm{p}<0.001$. C. Cell cycle status of tranduced cells analyzed by flow cytometry following PI staining presented as mean - s.d. (n=3) D-I. HL-60 cells +/- Cdh1-kd were treated with increasing concentrations of PMA for up to $48 \mathrm{~h}$. Expression of CD11b was analyzed by flow cytometry over time to follow differentiation and presented as mean $+/-$ s.d. $(\mathrm{D}+\mathrm{F} \mathrm{n}=6, \mathrm{H} n=3)$. Cells were sampled for immunoblotting to analyze Cdh1 and related target protein expression as indicated. $* \mathrm{p}<0.05, * * \mathrm{p}<0.01$. 
and higher p27 expression with further increase over time. Skp2 protein was assigned for degradation possibly due to ATRA-induced Cdh1 activation (Figure 3C), which only decreased at later time points when terminal granulocytic differentiation was already induced. The increase of the Cdk inhibitor p27 correlated well with cell cycle arrest in G0/G1 upon ATRA stimulation at 24 hours (Figure 3D). There was no significant difference on viability during the course of ATRA differentiation compared to the DMSO control (Figure 3E).

\section{ATRA could induce differentiation in APL independent of $\mathrm{Cdh} 1$ expression}

To further analyze the effect of Cdh1 in APL on ATRA-induced differentiation, we transduced NB4 cells with vector control and shRNAs against Cdh1 and Skp2. Cdh1-kd led to stabilization of its targets Aurora A and Skp2 without significant deregulation of p27 indicating functional kd, while Skp2-kd showed stabilization of its target p27 and also of Cdh1 (Figure 4A). Cdh1-kd led to
A

NB4

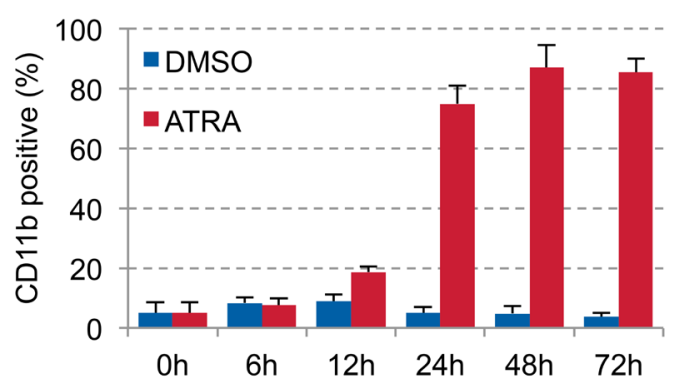

C

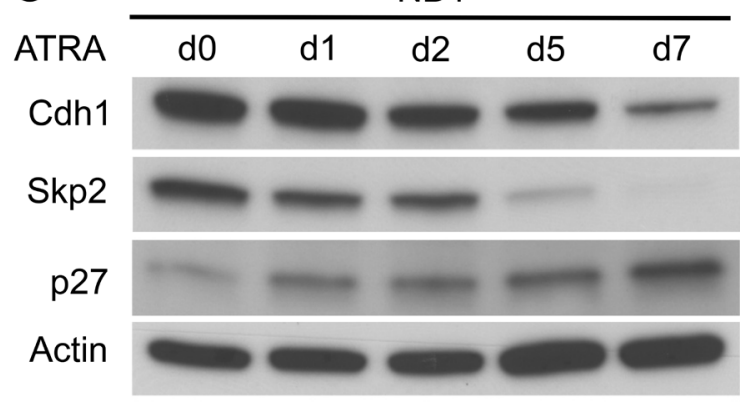

E

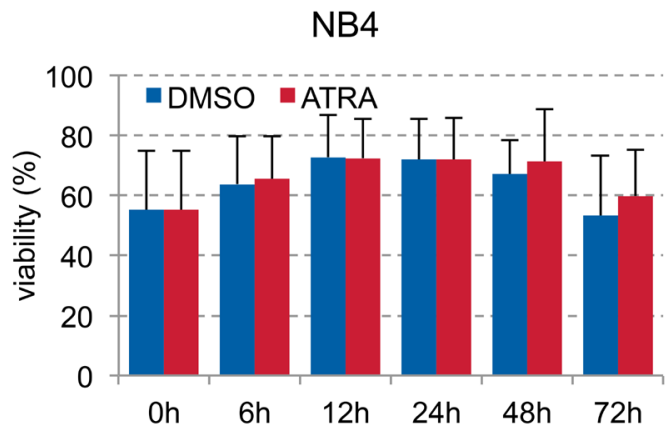

B

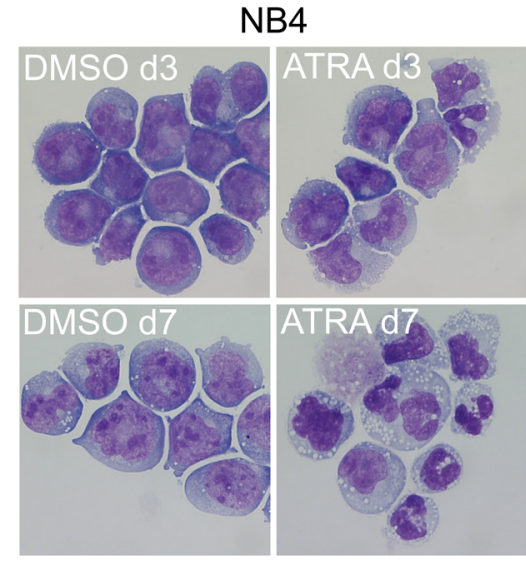

D

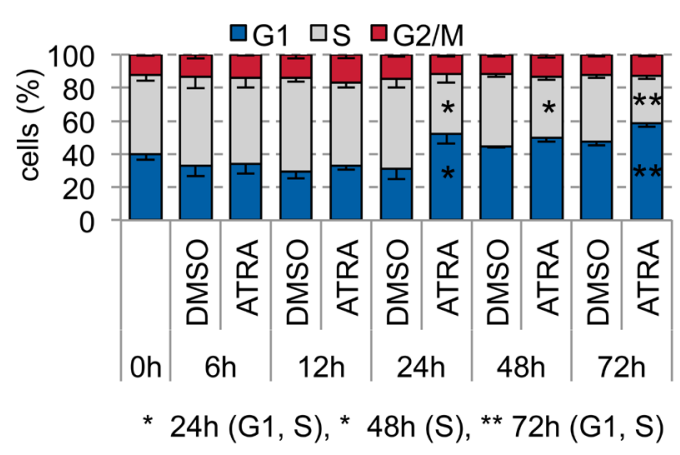

Figure 3: ATRA-induced granulocytic differentiation of APL cell line NB4. A. NB4 were plated at $0.2 \times 10^{6}$ cells $/ \mathrm{ml}$ and treated with $1 \mu \mathrm{M}$ ATRA or DMSO for $72 \mathrm{~h}$. Induction of CD11b expression to follow differentiation was analyzed by flow cytometry and presented as mean + s.d. $(n=3)$. B. Differential staining of NB4 cells at 3 and 7 days of ATRA treatment compared to DMSO control to visualize differentiation to granulocytes in ATRA treated cells. C. NB4 cells were cultured for up to 1 week in presence of $1 \mu \mathrm{M}$ ATRA and sampled for immunoblotting at the indicated time points. D. Cell cycle status of cells in (A) analyzed by flow cytometry following PI staining and presented as mean - s.d. $(\mathrm{n}=3)$. $* \mathrm{p}<0.05$, $* * \mathrm{p}<0.01$. E. Viability of the cells was determined by flow cytometry using dye exclusion of PI and presented as mean + s.d. $(n=3)$. 
increased proportion of $\mathrm{S}$ phase cells confirming previous results in other cancer cell lines [4, 31] compared to a decrease when Skp2 was depleted (Figure 4B). However, when we stimulated NB4 cells with ATRA we found that ATRA could rapidly induce differentiation (Figure 4C) and G0/G1 arrest (Figure 4D) equally well in cells with and without Cdh1-kd. This was independent of the ATRA concentration (Supplementary Figure S1) possibly due to strong transcriptional activation of inducers of differentiation which are no relevant $\mathrm{Cdh} 1$ targets and because APL biology depends mainly on $\mathrm{t}(15 ; 17)$. However, viability of NB4 cells with Cdh1-kd was significantly reduced upon ATRA stimulation over time (Figure 4E), suggesting that low Cdh1 expression contributes to ATRA-induced cell death.

To make results between HL-60 and NB4 more comparable, we also stimulated HL-60 with $1 \mu \mathrm{M}$ ATRA and NB4 with 1 nM PMA. ATRA had similar effects on HL-60 than PMA with significant lower expression of CD11b upon Cdh1-kd, which was preserved following stimulation with ATRA (Supplementary Figure S2B). There were also minimal, although significant differences on the very low CD14 expression following Cdh1-kd without further induction by ATRA stimulation. PMA induced rapid differentiation in NB4 cells without a significant difference in CD11b and/or CD14 expression
A

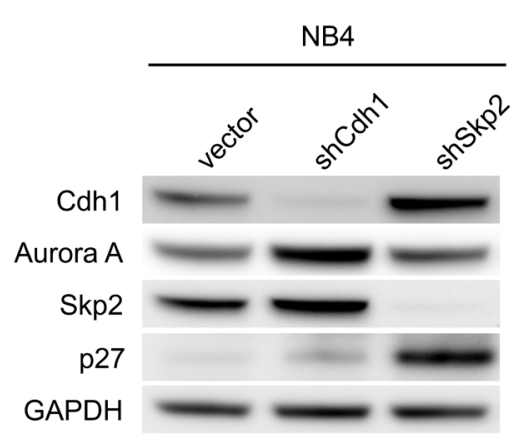

C

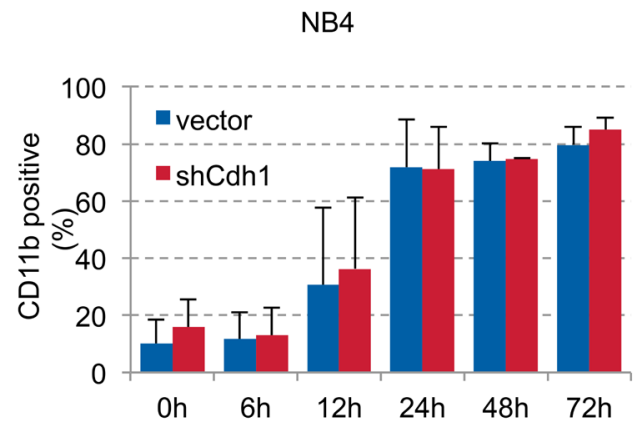

E

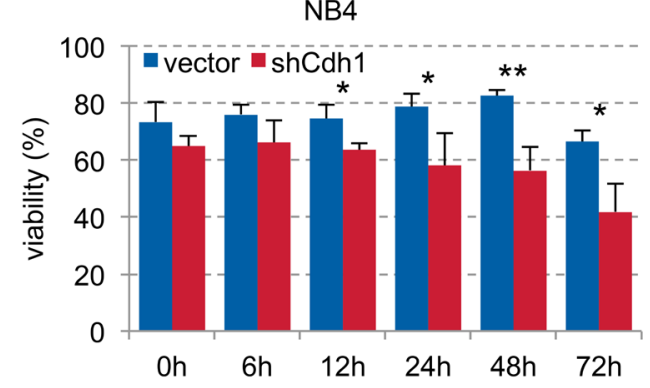

B

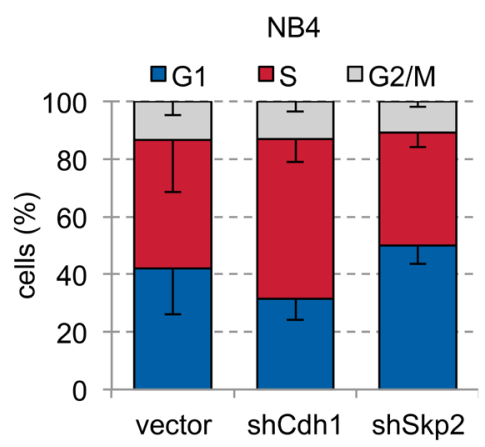

D

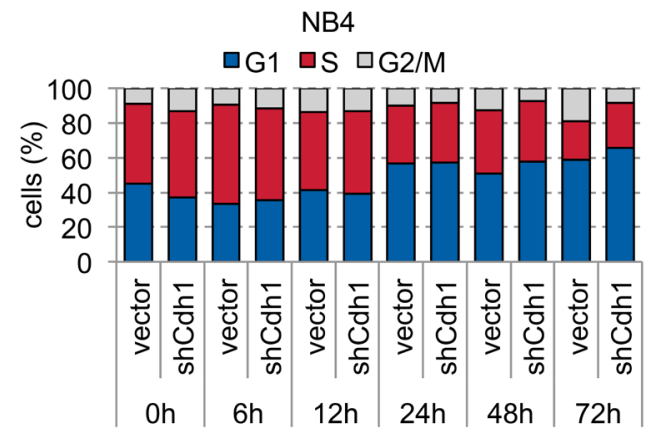

Figure 4: Influence of Cdh1-kd in NB4 cells on ATRA-induced granulocytic differentiation and viability. A. NB4 cells were transduced with constructs expressing shRNA specific for human Cdh1 and Skp2 and analyzed by western blot for indicated proteins. B. Cell cycle status of transduced NB4 cells was analyzed by flow cytometry following PI staining and presented as mean - s.d. ( $\mathrm{n}=3$ ). C-E. NB4 cells were transduced with Cdh1 knockdown or empty vector and treated with $1 \mu \mathrm{M}$ ATRA for up to $72 \mathrm{~h}$. Induction of CD11b to follow differentiation $(n=3)$, PI staining for cell cycle analysis $(0-48 h n=2,72 h n=1)$ and detection of viability $(n=3)$ was determined by flow cytometry and presented as mean + s.d. $(\mathrm{C}, \mathrm{E}) .{ }^{*} \mathrm{p}<0.05, * * \mathrm{p}<0.01$. 
+/- Cdh1-kd, which is comparable to the ATRA effect on NB4 (Supplementary Figure S2C). The depletion of Cdh1 did not change overall proliferation rate of HL-60 and NB4 cells (Supplementary Figure S2D).

\section{DISCUSSION}

Cdh1 is an important regulator of proliferation and differentiation. While we found Cdh1 expression decreased in the vast majority of primary AMLs of all subtypes, Cdh1 deficiency contributes to the differentiation block in AML with maturation but not in APL. This difference is relevant, because APL is, in contrast to all other AML subtypes, highly responsive to differentiation therapy with ATRA leading in combination with other compounds, such as anthracyclines or arsenic trioxide, to high complete remission rates of the disease and long-term survival. Moreover, while low Cdh1 expression in APL did not attenuate ATRA-induced differentiation, it enhances cell death and may thereby contribute to the therapeutic effect. APL harbors a unique chromosome translocation $\mathrm{t}(15 ; 17)$ resulting in the PMLRAR $\alpha$ fusion gene which may cause the accumulation of undifferentiated promyelocytes by constitutive repression of differentiation. This is counteracted by retinoic acid, which induces myeloid differentiation [32]. Thus, in APL the transcriptional repression of differentiation genes by PML-RAR $\alpha$ is mainly responsible for the differentiation block and modulation of $\mathrm{APC} / \mathrm{C}^{\mathrm{Cdh}} \mathrm{L}_{\text {- }}$ dependent protein ubiquitination is ineffective. In contrast, PMA induces differentiation by transcriptional regulation and enhancement of protein ubiquitination. It has been shown that expression of Cdh1 is specifically increased during PMA-induced megakaryocyte differentiation of the bcr-abl positive myeloid leukemia cell line K562 [33], hence supporting PMA effects in non-APL AML without $\mathrm{t}(15 ; 17)$, which is consistent with our data in HL-60. Cdh1-kd delays differentiation in HL-60 potentially by stabilizing Id 2 (inhibitor of differentiation) without significantly altering the cell cycle profile or proliferation (Figure 2C, Supplementary Figure S2). In NB4, in contrast, Cdh1-kd resulted in increased S phase cells without affecting differentiation. ATRA may induce proliferation and differentiation of APL cells leading to the accumulation of genetically unstable differentiated cells with increased apoptosis when Cdh1 expression is low. The combination of ATRA with anthracyclinechemotherapy is standard of care in high risk APL. Our previous results demonstrated that depletion of $\mathrm{Cdh} 1$ prolongs $\mathrm{S}$ phase, promotes replication stress and genomic instability and acts synergistically on doxorubicin-induced cell cycle arrest in $\mathrm{G} 2 / \mathrm{M}$ [25]. These observations provide an excellent explanation why the combination of ATRA und anthracyclines is highly efficient in (Cdh1 low) APL.

We found that Cdh1 is post-transcriptionally repressed in AML, which may be controlled by the
SCF ubiquitin ligase. It has recently been shown that $\mathrm{SCF}^{\text {BTrCP }}$ can ubiquitinate Cdh1 for degradation [28]. The overexpression of SCF subunit Skp2 has been shown to contribute to tumorigenesis in prostate cancer, breast cancer and lymphoma [34-37]. Since Skp2 protein expression is also increased in AML [29], we analyzed a potential additional role of Skp2 in Cdh1 degradation and found elevated Cdh1 levels in leukemia cells depleted of Skp2. This is an intriguing observation, since Skp2 is also a target of Cdh1. Thus, there may be an interesting feedback loop where early G1 is maintained by Cdh1mediated degradation of Skp2 and prevention of $\mathrm{p} 21 /$ p27 degradation. In late $\mathrm{G} 1$, when $\mathrm{Cdh} 1$ is inactivated by phosphorylation and its inhibitor Emi1, Skp2 becomes stable and can degrade p21, p27 and Cdh1 leading to S phase entry and cell cycle progression. Whether Cdh1 is a direct $\mathrm{SCF}^{\text {Skp2 }}$ target, remains to be further analyzed.

In summary, depending on differences in the microenvironment and the additional genetic background, our data are the first - to the best of our knowledge - to conclusively suggest that low Cdh1 expression may be important in AML biology by contributing to the differentiation block in non-APL AML and response to therapy in APL. It will be interesting to further analyze the function of Cdh1 in the differentiation and self-renewal of CD34+ normal hematopoietic and leukemic stem cells.

\section{MATERIALS AND METHODS}

\section{Patients}

We analyzed 29 samples of newly diagnosed acute myeloid leukemia (AML) patients at the Freiburg University Medical Center who gave written informed consent. Leukemic blasts were obtained from BM (17/29) and $\mathrm{PB}(12 / 29)$ specimens that contained high proportions of blast cells by Ficoll density gradient centrifugation. Detailed patient characteristics are summarized in Table 1. Normal CD34+ cells from mobilized autologous donors who gave informed consent were purified from apheresis by immunomagnetic cell separation Kit (CD34 MicroBead Kit, Miltenyi) [38].

\section{Microarray analysis}

Microarray datasets were obtained from the openaccess NIH Gene-Expression Omnibus (GEO) database. Datasets used in our analysis were GSE30029 [26].

\section{Western blot and antibodies}

Immunoblotting has been performed as previously described [4]. Antibodies used were anti-Cdh1 (Merck, Abcam), anti-Cullin1 (Abcam), anti-Skp2 (Cell Signaling, Santa Cruz), anti-p27 (BD Bioscience, Cell Signaling), anti-Id2 (Santa Cruz), anti-Aurora A (BD Bioscience), 
anti-GAPDH (GeneTex) anti-Actin (Sigma) and horseradish-peroxidase-conjugated anti-mouse (Sigma, Dako) and anti-rabbit (Amersham) secondary antibodies. For densitometry of Western blots, the Gel iX imager (Intas Science Imaging Instruments, Göttingen, Germany) and LabImage 1D (Intas) were used.

\section{FACS analysis}

Cells were labelled with RPE- or FITC-conjugated anti-CD11b antibody (Dako, AbD Serotech). As an isotype control Simultest Control $\gamma 1 / \gamma 1$ (BD Pharmingen) was used. Propidium iodide staining for cell cycle analyses was performed as described previously [4]. Cells were analysed on a FACS Calibur (BD Bioscience, Franklin Lakes, USA) using the Cellquest software (BD Bioscience, Franklin Lakes, USA) or on LSR Fortessa (BD) and FACS Diva 6.2 software for acquisition. For data analysis FlowJo 7.6.5 was used.

\section{Plasmids}

For lentiviral knockdown of Cdh1, Skp2 and GFP in HL-60 cells a previously described pLentiLox3.7 vector was used [4]. Oligonucleotides (Apara Bioscience $\mathrm{GmbH}$ ) containing a 19 bp shRNA directed against Cdh1 (5'-GGATTAACGAGAATGAGAA-3') and GFP (5'-GGCATCAAGGTGAACTTCA-3'), designed by our laboratory [4], and Skp2 (5'-GAGGAGCCCGACAGTGAGA-3') [37], were annealed and ligated into HpaI/XhoI-site of pLentiLox3.7. Experiments in NB4 cells were carried out with the pLeGOhU6-G expression vector [39, 40]. The selection marker GFP was exchanged with RFP using the BamHI and EcoRI restriction sites. Oligonucleotides against Cdh1 and Skp2 were annealed and ligated into HpaI/XhoI-site of pLeGOhU6-RFP as described to obtain pLeGOhU6-RFPCdh1-kd. pLeGOhU6-RFP served as a control. A dominant negative Cullin1 N252 (Cul1-N252) construct that disrupts the function of the SCF complex was obtained from D. Guardavaccaro (Utrecht). The Cul1-N252 fragment was subcloned into pLeGO-iG [39] using BamHI and NotI restriction sites.

\section{Cell culture and lentiviral transduction}

Cell lines Kasumi-1, HL-60 (gift from M. Lübbert) and NB4 (DSMZ \#ACC 207) were cultured in RPMI 1640 medium (with $2 \mathrm{mM}$ L-Glutamine, Gibco) supplemented with $10 \%$ fetal bovine serum (Biochrom), $1 \%$ penicillin/ streptomycin (Life Technologies) and $1 \mathrm{mM}$ sodium pyruvate (Life Technologies). Suspension cells were kept at a density between $0.1-0.5 \times 10^{6}$ cells $/ \mathrm{ml}$. Lentiviral infections of cell lines were performed as previously described [4].

\section{Reagents}

Phorbol-12-myristate-13-acetate (PMA, Sigma) was dissolved to $50 \mu \mathrm{M}$ in DMSO/Ethanol (50\% each). Up to $50 \mathrm{nM}$ PMA was added to the culture, while the final DMSO concentration did not exceeded $0.1 \%$. All-trans retinoic acid (ATRA, Sigma) was dissolved in DMSO to receive $50 \mathrm{mM}$ stock solution.

\section{Differential staining (Pappenheim's stain)}

Cells were spun onto glass slides by cytocentrifugation and stained for $5 \mathrm{~min}$ in MayGruenwald's-eosin-methylene blue solution (Merck). After washing in PBS for 3 min slides were placed in diluted (1:20 in PBS) Giemsa's azur eosin methylene blue solution (Merck) for $20 \mathrm{~min}$. Staining was stopped by washing with destilled water. Slides were imaged using an Axio Imager. A2 (Zeiss) with Plan Apochromat 63x/1.4 oil immersion objective.

\section{Statistical analysis}

A two-sided Student's t-test was used for statistical analyses. Mean + standard deviation (s.d.) were ploted as indicated. P-values were defined as indicated in the figure legends $* \mathrm{p}<0.05, * * \mathrm{p}<0.01, * * * \mathrm{p}<0.001$.

\section{ACKNOWLEDGMENTS}

We thank D. Guardavaccaro for providing plasmid and J. Felthaus and D. Wider for excellent technical assistance.

\section{CONFLICTS OF INTEREST}

The authors declare no conflicts of interest.

\section{FUNDING}

This work is supported by Jose-Carreras Leukemia Foundation.

\section{REFERENCES}

1. Schnerch D, Yalcintepe J, Schmidts A, Becker H, Follo M, Engelhardt M, Wäsch R. Cell cycle control in acute myeloid leukemia. American journal of cancer research. 2012; 2:508-528.

2. Schnerch D, Schmidts A, Follo M, Udi J, Felthaus J, Pfeifer D, Engelhardt M, Wäsch R. BubR1 is frequently repressed in acute myeloid leukemia and its re-expression sensitizes cells to antimitotic therapy. Haematologica. 2013; 98:1886-1895.

3. Garcia-Higuera I, Manchado E, Dubus P, Canamero M, Mendez J, Moreno S, Malumbres M. Genomic stability and 
tumour suppression by the APC/C cofactor Cdh1. Nat Cell Biol. 2008; 10:802-811.

4. Engelbert D, Schnerch D, Baumgarten A, Wäsch R. The ubiquitin ligase $\mathrm{APC}(\mathrm{Cdh} 1)$ is required to maintain genome integrity in primary human cells. Oncogene. 2008; 27:907-917.

5. Eguren M, Porlan E, Manchado E, Garcia-Higuera I, Canamero M, Farinas I, Malumbres M. The APC/C cofactor Cdh1 prevents replicative stress and p53-dependent cell death in neural progenitors. Nature communications. 2013; $4: 2880$.

6. Delgado-Esteban M, Garcia-Higuera I, Maestre C, Moreno $\mathrm{S}$, Almeida A. APC/C-Cdh1 coordinates neurogenesis and cortical size during development. Nature communications. 2013; 4:2879.

7. Stegmüller J, Konishi Y, Huynh MA, Yuan Z, Dibacco S, Bonni A. Cell-intrinsic regulation of axonal morphogenesis by the Cdh1-APC target SnoN. Neuron. 2006; 50:389-400.

8. Lasorella A, Stegmuller J, Guardavaccaro D, Liu G, Carro MS, Rothschild G, de la Torre-Ubieta L, Pagano M, Bonni A, Iavarone A. Degradation of Id 2 by the anaphasepromoting complex couples cell cycle exit and axonal growth. Nature. 2006; 442:471-474.

9. Wäsch R, Robbins JA, Cross FR. The emerging role of $\mathrm{APC} / \mathrm{CCdh} 1$ in controlling differentiation, genomic stability and tumor suppression. Oncogene. 2010; 29:1-10.

10. Wäsch R, Cross FR. APC-dependent proteolysis of the mitotic cyclin $\mathrm{Clb} 2$ is essential for mitotic exit. Nature. 2002; 418:556-562.

11. Sigrist SJ, Lehner CF. Drosophila fizzy-related downregulates mitotic cyclins and is required for cell proliferation arrest and entry into endocycles. Cell. 1997; 90:671-681.

12. Blanco MA, Sanchez-Diaz A, de Prada JM, Moreno S. APC(ste9/srw1) promotes degradation of mitotic cyclins in $\mathrm{G}(1)$ and is inhibited by cdc2 phosphorylation. EMBO J. 2000; 19:3945-3955.

13. Yamaguchi S, Okayama H, Nurse P. Fission yeast Fizzyrelated protein srw1p is a $\mathrm{G}(1)$-specific promoter of mitotic cyclin B degradation. EMBO J. 2000; 19:3968-3977.

14. Bashir T, Dorrello NV, Amador V, Guardavaccaro D, Pagano M. Control of the SCF(Skp2-Cks1) ubiquitin ligase by the $\mathrm{APC} / \mathrm{C}(\mathrm{Cdh} 1)$ ubiquitin ligase. Nature. 2004; 428:190-193.

15. Wei W, Ayad NG, Wan Y, Zhang GJ, Kirschner MW, Kaelin WG, Jr. Degradation of the SCF component Skp2 in cell-cycle phase G1 by the anaphase-promoting complex. Nature. 2004; 428:194-198.

16. Wirth KG, Ricci R, Gimenez-Abian JF, Taghybeeglu S, Kudo NR, Jochum W, Vasseur-Cognet M, Nasmyth K. Loss of the anaphase-promoting complex in quiescent cells causes unscheduled hepatocyte proliferation. Genes Dev. 2004; 18:88-98.
17. Stroschein SL, Bonni S, Wrana JL, Luo K. Smad3 recruits the anaphase-promoting complex for ubiquitination and degradation of SnoN. Genes Dev. 2001; 15:2822-2836.

18. Wan Y, Liu X, Kirschner MW. The anaphase-promoting complex mediates TGF-beta signaling by targeting SnoN for destruction. Mol Cell. 2001; 8:1027-1039.

19. Hu D, Wan Y. Regulation of Kruppel-like factor 4 by the anaphase promoting complex pathway is involved in TGFbeta signaling. J Biol Chem. 2011; 286:6890-6901.

20. Gieffers C, Peters BH, Kramer ER, Dotti CG, Peters JM. Expression of the CDH1-associated form of the anaphasepromoting complex in postmitotic neurons. Proc Natl Acad Sci U S A. 1999; 96:11317-11322.

21. Konishi Y, Stegmuller J, Matsuda T, Bonni S, Bonni A. Cdh1-APC controls axonal growth and patterning in the mammalian brain. Science. 2004; 303:1026-1030.

22. Li W, Wu G, Wan Y. The dual effects of Cdh1/APC in myogenesis. Faseb J. 2007; 21:3606-3617.

23. Wu G, Glickstein S, Liu W, Fujita T, Li W, Yang Q, Duvoisin R, Wan Y. The anaphase-promoting complex coordinates initiation of lens differentiation. Mol Biol Cell. 2007; 18:1018-1029.

24. Bar-On O, Shapira M, Skorecki K, Hershko A, Hershko DD. Regulation of $\mathrm{APC} / \mathrm{C}(\mathrm{Cdh} 1)$ ubiquitin ligase in differentiation of human embryonic stem cells. Cell Cycle. $2010 ; 9$.

25. Greil C, Krohs J, Schnerch D, Follo M, Felthaus J, Engelhardt M, Wäsch R. The role of $\mathrm{APC} / \mathrm{CCdh} 1$ in replication stress and origin of genomic instability. Oncogene. 2015 Oct 12. doi: 10.1038/onc.2015.367. [Epub ahead of print]

26. de Jonge HJ, Woolthuis CM, Vos AZ, Mulder A, van den Berg E, Kluin PM, van der Weide K, de Bont ES, Huls G, Vellenga E, Schuringa JJ. Gene expression profiling in the leukemic stem cell-enriched CD34+ fraction identifies target genes that predict prognosis in normal karyotype AML. Leukemia. 2011; 25:1825-1833.

27. Benmaamar R, Pagano M. Involvement of the SCF complex in the control of Cdh1 degradation in S-phase. Cell Cycle. $2005 ; 4: 1230-1232$.

28. Fukushima H, Ogura K, Wan L, Lu Y, Li V, Gao D, Liu P, Lau AW, Wu T, Kirschner MW, Inuzuka H, Wei W. SCFmediated Cdh1 degradation defines a negative feedback system that coordinates cell-cycle progression. Cell reports. 2013; 4:803-816.

29. Min YH, Cheong JW, Lee MH, Kim JY, Lee ST, Hahn JS, Ko YW. Elevated S-phase kinase-associated protein 2 protein expression in acute myelogenous leukemia: its association with constitutive phosphorylation of phosphatase and tensin homologue protein and poor prognosis. Clin Cancer Res. 2004; 10:5123-5130.

30. Dalton WT Jr, Ahearn MJ, McCredie KB, Freireich EJ, Stass SA, Trujillo JM. HL-60 cell line was derived from 
a patient with FAB-M2 and not FAB-M3. Blood. 1988; 71:242-7.

31. Sigl R, Wandke C, Rauch V, Kirk J, Hunt T, Geley S. Loss of the mammalian APC/C activator FZR1 shortens G1 and lengthens $\mathrm{S}$ phase but has little effect on exit from mitosis. J Cell Sci. 2009; 122:4208-4217.

32. Lo-Coco F, Hasan SK. Understanding the molecular pathogenesis of acute promyelocytic leukemia. Best practice \& research Clinical haematology. 2014; 27:3-9.

33. Li C, Peart N, Xuan Z, Lewis DE, Xia Y, Jin J. PMA induces SnoN proteolysis and CD61 expression through an autocrine mechanism. Cellular signalling. 2014; 26:1369-1378.

34. Ben-Izhak O, Lahav-Baratz S, Meretyk S, Ben-Eliezer S, Sabo E, Dirnfeld M, Cohen S, Ciechanover A. Inverse relationship between Skp2 ubiquitin ligase and the cyclin dependent kinase inhibitor p27Kip1 in prostate cancer. J Urol. 2003; 170:241-245.

35. Latres E, Chiarle R, Schulman BA, Pavletich NP, Pellicer A, Inghirami G, Pagano M. Role of the F-box protein Skp2 in lymphomagenesis. Proc Natl Acad Sci U S A. 2001; 98:2515-2520.

36. Signoretti S, Di Marcotullio L, Richardson A, Ramaswamy S, Isaac B, Rue M, Monti F, Loda M, Pagano M. Oncogenic role of the ubiquitin ligase subunit Skp2 in human breast cancer. J Clin Invest. 2002; 110:633-641.

37. Fujita T, Liu W, Doihara H, Date H, Wan Y. Dissection of the APCCdh1-Skp2 cascade in breast cancer. Clin Cancer Res. 2008; 14:1966-1975.

38. Engelhardt M, Mackenzie K, Drullinsky P, Silver RT, Moore MA. Telomerase activity and telomere length in acute and chronic leukemia, pre- and post-ex vivo culture. Cancer Res. 2000; 60:610-617.

39. Weber K, Bartsch U, Stocking C, Fehse B. A multicolor panel of novel lentiviral "gene ontology" (LeGO) vectors for functional gene analysis. Mol Ther. 2008; 16:698-706.

40. Roelz R, Pilz IH, Mutschler M, Pahl HL. Of mice and men: human RNA polymerase III promoter U6 is more efficient than its murine homologue for shRNA expression from a lentiviral vector in both human and murine progenitor cells. Exp Hematol. 2010; 38:792-797. 